\title{
Green's Function and Positive Solutions for a Second-Order Singular Boundary Value Problem with Integral Boundary Conditions and a Delayed Argument
}

\author{
Xuemei Zhang ${ }^{1}$ and Meiqiang Feng ${ }^{2}$ \\ ${ }^{1}$ Department of Mathematics and Physics, North China Electric Power University, Beijing 102206, China \\ ${ }^{2}$ School of Applied Science, Beijing Information Science \& Technology University, Beijing 100192, China \\ Correspondence should be addressed to Xuemei Zhang; zxm74@sina.com
}

Received 16 May 2014; Revised 15 July 2014; Accepted 16 July 2014; Published 6 August 2014

Academic Editor: Dumitru Baleanu

Copyright ( $) 2014$ X. Zhang and M. Feng. This is an open access article distributed under the Creative Commons Attribution License, which permits unrestricted use, distribution, and reproduction in any medium, provided the original work is properly cited.

\begin{abstract}
This paper investigates the expression and properties of Green's function for a second-order singular boundary value problem with integral boundary conditions and delayed argument $-x^{\prime \prime}(t)-a(t) x^{\prime}(t)+b(t) x(t)=\omega(t) f(t, x(\alpha(t))), t \in(0,1) ; x^{\prime}(0)=$ $0, x(1)-\int_{0}^{1} h(t) x(t) d t=0$, where $a \in([0,1],[0,+\infty)), b \in C([0,1],(0,+\infty))$ and, $\omega$ may be singular at $t=0$ or/and at $t=1$. Furthermore, several new and more general results are obtained for the existence of positive solutions for the above problem by using Krasnosel'skii's fixed point theorem. We discuss our problems with a delayed argument, which may concern optimization issues of some technical problems. Moreover, the approach to express the integral equation of the above problem is different from earlier approaches. Our results cover a second-order boundary value problem without deviating arguments and are compared with some recent results.
\end{abstract}

\section{Introduction}

Boundary value problems with integral boundary conditions arise naturally in thermal conduction problems [1], semiconductor problems [2], hydrodynamic problems [3], and so on. It is interesting to point out that such problems include two-, three-, and multipoint and nonlocal boundary value problems as special cases and have been extensively studied in the last ten years; see, for example, [4-18]. Recently, Feng et al. [19] applied the fixed point theory in a cone for strict set contraction operators to study the existence and multiplicity of positive solutions for the problem given by

$$
\begin{gathered}
x^{\prime \prime}+f(t, x)=\theta, \quad t \in(0,1), \\
x(0)=\theta, \quad x(1)=\int_{0}^{1} g(t) x(t) d t,
\end{gathered}
$$

where $\theta$ is the zero element of a real Banach space $E$.

At the same time, a class of boundary value problems with deviating arguments are receiving much attention. For example, in [20], Yang et al. studied the existence and multiplicity of positive solutions to a three-point boundary value problem with an advanced argument:

$$
\begin{gathered}
x^{\prime \prime}(t)+a(t) f(x(\alpha(t)))=0, \quad t \in(0,1), \\
x(0)=0, \quad b x(\eta)=x(1),
\end{gathered}
$$

where $0<\eta<1, b>0$, and $1-b \eta>0$. The main tool is the fixed point index theory. It is clear that the solution of [20] is concave when $a(t) \geq 0$ on $[0,1]$ and $f(x) \geq 0$ on $[0, \infty)$. However, few papers have reported the same problems where the solution is without concavity; for example, see some recent excellent results and applications of the case of ordinary differential equations with deviating arguments to a variety of problems from Jankowski [21-23], Jiang and Wei [24], Wang [25], Wang et al. [26], and Hu et al. [27]. This paper will resolve this problem. 
Consider the second-order singular boundary value problem with integral boundary conditions and a delayed argument:

$$
\begin{gathered}
L x=\omega(t) f(t, x(\alpha(t))), \quad t \in(0,1), \\
x^{\prime}(0)=0, \quad x(1)-\int_{0}^{1} h(t) x(t) d t=0,
\end{gathered}
$$

where $L$ denotes the linear operator and

$$
L x:=-x^{\prime \prime}-a x^{\prime}+b x
$$

where $a \in C([0,1],[0,+\infty)), b \in C([0,1],(0,+\infty)), f \in$ $C([0,1] \times[0,+\infty) \rightarrow[0,+\infty))$, and $\omega$ may be singular at $t=0$ or/and at $t=1$.

Throughout this paper, we assume that $\alpha(t) \not \equiv t$ on $J=$ $[0,1]$. In addition, $\omega, f, \alpha$, and $h$ satisfy the following:

$\left(H_{1}\right) \omega \in C((0,1),[0,+\infty))$ with $0<\int_{0}^{1} \omega(s) d s<\infty$ and $\omega$ does not vanish on any subinterval of $(0,1)$;

$\left(H_{2}\right) f \in C([0,1] \times[0,+\infty),[0,+\infty)), \alpha \in C(J, J)$ with $\alpha(t) \leq t$ on $J$

$\left(H_{3}\right) h \in C[0,1]$ is nonnegative with $\nu \in[0,1)$, where

$$
v=\int_{0}^{1} h(t) \phi(t) d t
$$

where $\phi$ satisfies

$$
-\phi^{\prime \prime}(t)-a \phi^{\prime}(t)+b \phi(t)=0, \quad \phi^{\prime}(0)=0, \phi(1)=1 .
$$

Remark 1. Generally, when $y(t) \geq 0$ on $J$, the solution $x$ is not concave for the linear equation

$$
L x-y(t)=0 \text {. }
$$

This means that the method depending on concavity is no longer valid, and we need to introduce a new method to study this kind of problems.

Remark 2. For simplicity we only consider Neumann boundary conditions since all the results obtained in this paper can also be adapted with minor changes to the other boundary conditions.

Some special cases of (3), such as boundary value problems with delay, have been investigated [28-32]. It is not difficult to see that the corresponding function $f$ appearing on the right-hand side depends on $x(t-\tau), \tau>0$, where initial function $x$ is given on the initial set, for example, $[-\tau, 0]$. T. Jankowski and R. Jankowski [33, 34] pointed out that, in such cases $\alpha(t)=t-\tau$, there are some problems with a constant delay $\tau$. If we consider the differential problem on intervals $[0, k]$, where $k \leq \tau$, then it means that we have no delays; we have such a situation in [32]. If $k>\tau$, then it is easy to solve the differential equation on $[0, \tau]$, since we have the solution on the initial set $[-\tau, 0]$. Continuing this process, we can find a solution on the whole interval $[0, k]$, by using the method of steps. In our paper, for example, the deviating argument $\alpha$ can have a form $\alpha(t)=\rho t=t-(1-\rho) t$ with a fixed number $\rho \in(0,1)$, so the delay $(1-\rho) t$ is a function of $t$. In this case, the initial set reduces to one point $t=0$, and we cannot apply the step method. To our knowledge, it is the first paper in which positive solution has been investigated for a secondorder singular differential equation with a delayed argument under the case that $L x:=-x^{\prime \prime}-a x^{\prime}+b x$.

Being directly inspired by $[5,12,20,21]$, the authors will prove several new and more general results for the existence of positive solutions for problem (3) by using fixed point theories in a cone. Another contribution of this paper is to study the expression and properties of Green's function associated with problem (3). The expression of the integral equation is simpler than that of $[5,12]$.

The organization of this paper is as follows. In Section 2, we present the expression and properties of Green's function associated with the problem (3). In Section 3, we present some definitions and lemmas which are needed throughout this paper. In Section 4, we use fixed point theorem to obtain the existence of positive solutions for problem (3) with a delayed argument $\alpha$. In particular, our results in these sections are new when $\alpha(t) \equiv t$ on $t \in J$. Finally, in Section 5, three examples are also included to illustrate the main results.

\section{Expression and Properties of Green's Function}

Theorem 3. Assume that $v \neq 1$. Then for any $y \in C[0,1]$, the boundary value problem

$$
\begin{gathered}
-x^{\prime \prime}(t)-a(t) x^{\prime}(t)+b(t) x(t)-y(t)=0, \quad t \in(0,1), \\
x^{\prime}(0)=0, \quad x(1)-\int_{0}^{1} h(t) x(t) d t=0
\end{gathered}
$$

has a unique solution

$$
x(t)=\int_{0}^{1} H(t, s) q(s) y(s) d s,
$$

where

$$
\begin{gathered}
q(t)=\exp \left(\int_{0}^{t} a(s) d s\right), \quad H(t, s)=G(t, s)+G_{1}(t, s), \\
G(t, s)=\frac{1}{\Delta} \begin{cases}\phi(s) \psi(t), & \text { if } 0 \leq s \leq t \leq 1, \\
\phi(t) \psi(s), & \text { if } 0 \leq t \leq s \leq 1,\end{cases} \\
G_{1}(t, s)=\frac{\phi(t)}{1-v} \int_{0}^{1} G(\tau, s) h(\tau) d \tau .
\end{gathered}
$$

Here $\phi$ and $\psi$ satisfy (6) and

$$
L \psi=0, \quad \psi(0)=1, \quad \psi(1)=0,
$$

respectively.

Proof. The proof is similar to that of Lemma 2.3 in [5] and Lemma 2.1 in [35]. 
Remark 4. It is not difficult from $[5,12]$ to show that $\Delta:=$ $-\phi(0) \psi^{\prime}(0)>0$ and (i) $\phi$ is nondecreasing on $J$ and $\phi>0$ on $J$ and (ii) $\psi$ is strictly decreasing on $J$.

Remark 5. The expression of the integral equation (9) is different from that of (2.10) in [5] and that of (2.9) in [12].

Remark 6. Noticing $a \in C([0,1],[0,+\infty))$, it follows from the definition of $q$ that

$$
1 \leq q(t) \leq e^{M} \text { for } t \in J
$$

where $M=\max _{t \in J} a(t)$.

Lemma 7. Let $\xi \in(0,1), G, G_{1}$, and $H$ be given as in Theorem 3. Then one has the following results:

$$
\begin{gathered}
G(t, s) \leq G(s, s), \quad G_{1}(t, s) \leq G(1, s), \\
H(t, s) \leq H(s) \leq H^{0}, \\
\forall t, s \in J, \\
G(t, s) \geq \delta G(s, s), \quad G_{1}(t, s) \geq \phi(0) G_{1}(1, s), \\
H(t, s) \geq \delta H(s) \geq \delta H_{0}, \\
\forall t \in[0, \xi], \quad s \in J,
\end{gathered}
$$

where

$$
\begin{gathered}
H(s)=G(s, s)+G_{1}(1, s), \quad H^{0}=\max _{s \in J} H(s), \\
H_{0}=\min _{s \in J} H(s), \quad \delta=\min \{\psi(\xi), \phi(0)\} .
\end{gathered}
$$

Proof. Noticing Remark 4, it follows from the definition of $G(t, s), G_{1}(t, s)$, and $H(t, s)$ that (13) holds. Now, we show that (14) also holds.

In fact, for $t \in[0, \xi]$ and $s \in J$, we have

$$
\begin{aligned}
\frac{G(t, s)}{G(s, s)} & \geq \min \left\{\frac{\psi(t)}{\psi(s)}, \frac{\phi(t)}{\phi(s)}\right\} \geq \min \left\{\frac{\psi(0)}{\psi(0)}, \frac{\phi(\xi)}{\phi(1)}\right\} \\
& =\{\psi(\xi), \phi(0)\}=: \delta .
\end{aligned}
$$

Similarly, we can prove that $G_{1}(t, s) \geq G_{1}(0, s) \geq$ $\phi(0) G_{1}(1, s)$ for $t \in[0, \xi]$ and $s \in J$. This and (15) imply that

$$
\begin{array}{r}
H(t, s) \geq \delta G(s, s)+\phi(0) G_{1}(1, s)=\delta H(s), \\
\forall t \in[0, \xi], \quad s \in J .
\end{array}
$$

This gives the proof of Lemma 7 .

Remark 8. It follows from (13) and (14) that

$$
\delta H_{0} \leq H(t, s) \leq H^{0}, \quad \forall t \in[0, \xi], s \in J .
$$

\section{Preliminaries}

In this section, we first present some definitions and lemmas which are needed throughout this paper.
Definition 9 (see [36]). Let $E$ be a real Banach space over $\mathbb{R}$. A nonempty closed set $P \subset E$ is said to be a cone provided that

(i) $a u+b v \in P$ for all $u, v \in P$ and all $a \geq 0, b \geq 0$;

(ii) $u,-u \in P$ implies $u=0$.

Every cone $P \subset E$ induces an ordering in $E$ given by $x \leq y$ if and only if $y-x \in P$.

Let $E=C[0,1]$. Then $E$ is a real Banach space with the norm $\|\cdot\|$ defined by

$$
\|x\|=\max _{t \in J}|x(t)|, \quad x \in E .
$$

Definition 10. A function $x \in E \cap C^{2}(0,1)$ is called a solution of (3) if it satisfies (3). If $x(t) \geq 0$ and $x(t) \neq \equiv$ on $J$, then $x$ is called a positive solution of (3).

Define a cone $K$ in $E$ by

$$
K=\left\{x \in E: x(t) \geq 0, \min _{t \in[0, \xi]} x(t) \geq \delta\|x\|\right\} .
$$

Also, define, for a positive number $r, \Omega_{r}$ by

$$
\Omega_{r}=\{x \in E:\|x\|<r\} .
$$

Note that $\partial \Omega_{r}=\{x \in E:\|x\|=r\}$.

Define $T: K \rightarrow K$ by

$$
(T x)(t)=\int_{0}^{1} H(t, s) q(s) \omega(s) f(s, x(\alpha(s))) d s .
$$

Lemma 11. Assume that $\left(H_{1}\right)-\left(H_{3}\right)$ hold. Then, $T(K) \subset K$ and $T: K \rightarrow K$ are completely continuous.

Proof. For $x \in K$, it follows from (13) and (22) that

$$
\begin{aligned}
\|T x\| & =\max _{t \in J} \int_{0}^{1} H(t, s) q(s) \omega(s) f(s, x(\alpha(s))) d s \\
& \leq \int_{0}^{1} H(s) q(s) \omega(s) f(s, x(\alpha(s))) d s .
\end{aligned}
$$

It follows from (14), (22), and (23) that

$$
\begin{aligned}
\min _{t \in[0, \xi]}(T x)(t) & =\min _{t \in[0, \xi]} \int_{0}^{1} H(t, s) q(s) \omega(s) f(s, x(\alpha(s))) d s \\
& \geq \delta \int_{0}^{1} H(s) q(s) \omega(s) f(s, x(\alpha(s))) d s \\
& \geq \delta\|T x\| .
\end{aligned}
$$

Thus, $T(K) \subset K$.

Next, by standard methods and Ascoli-Arzela theorem, one can prove that $T: K \rightarrow K$ is completely continuous. So it is omitted, and the lemma is proved.

Remark 12. From (22), we know that $x \in E$ is a solution of problem (3) if and only if $x$ is a fixed point of operator $T$.

In the rest of this section, we state a well known fixed point theorem which we need later. 
Lemma 13 (see [36]). Let $P$ be a cone in a real Banach space E. Assume $\Omega_{1}$ and $\Omega_{2}$ are bounded open sets in $E$ with $0 \in \Omega_{1}$, $\bar{\Omega}_{1} \subset \Omega_{2}$. If

$$
A: P \cap\left(\bar{\Omega}_{2} \backslash \Omega_{1}\right) \longrightarrow P
$$

is completely continuous such that either

(i) $\|A x\| \leq\|x\|$, for all $x \in P \cap \partial \Omega_{1}$, and $\|A x\| \geq\|x\|$, for all $x \in P \cap \partial \Omega_{2}$, or

(ii) $\|A x\| \geq\|x\|$, for all $x \in P \cap \partial \Omega_{1}$, and $\|A x\| \leq\|x\|$, for all $x \in P \cap \partial \Omega_{2}$,

then $A$ has at least one fixed point in $P \cap\left(\bar{\Omega}_{2} \backslash \Omega_{1}\right)$.

\section{Existence of Single}

\section{or Twin Positive Solutions}

For convenience, we introduce the following notations:

$$
\begin{aligned}
& f^{0}=\lim _{y \rightarrow 0} \sup _{t \in J} \frac{f(t, y)}{y}, \quad f_{0}=\liminf _{y \rightarrow 0} \min _{t \in J} \frac{f(t, y)}{y}, \\
& f^{\infty}=\lim _{y \rightarrow \infty} \sup _{t \in J} \frac{f(t, y)}{y}, \quad f_{\infty}=\liminf _{y \rightarrow \infty} \min _{t \in J} \frac{f(t, y)}{y} .
\end{aligned}
$$

We also define as [37] $i_{0}=$ number of zeros in the set $\left\{f^{0}, f^{\infty}\right\}$ and $i_{\infty}=$ number of infinities in the set $\left\{f_{0}, f_{\infty}\right\}$. Sun and $\mathrm{Li}$ [38] pointed out that $i_{0}, i_{\infty}=0,1$, or 2 , and there are six possible cases: (i) $i_{0}=0$ and $i_{\infty}=0$; (ii) $i_{0}=0$ and $i_{\infty}=1$; (iii) $i_{0}=0$ and $i_{\infty}=2$; (iv) $i_{0}=1$ and $i_{\infty}=0$; (v) $i_{0}=1$ and $i_{\infty}=1$; and (vi) $i_{0}=2$ and $i_{\infty}=0$. By using Krasnoseliis fixed point theorem in a cone, some results are obtained for the existence of at least one or two positive solutions of problem (3) for $\alpha(t) \leq t$ on $J$ under the above six possible cases.

4.1. For the Case $i_{0}=1$ and $i_{\infty}=1$. In this subsection, we discuss the existence of single positive solution for problem (3) under $i_{0}=1$ and $i_{\infty}=1$.

For convenience, we introduce the following notations:

$$
\gamma=\int_{0}^{1} \omega(s) d s, \quad \gamma_{1}=\int_{\xi}^{1} \omega(s) d s .
$$

Theorem 14. Assume that $\left(H_{1}\right)-\left(H_{3}\right)$ hold. If $i_{0}=1$ and $i_{\infty}=$ 1 , then problem (3) has at least one positive solution.

Proof. First, we consider the case $f^{0}=0$ and $f_{\infty}=\infty$. Since $f^{0}=0$, then there exists $r>0$ such that

$$
f(t, x) \leq \frac{1}{H^{0} \gamma e^{M}} x \quad \forall t \in J, 0 \leq x \leq r .
$$

Since $0 \leq \alpha(t) \leq t \leq 1$ on $[0, \xi]$, it follows from $0 \leq x(t) \leq$ $r$ on $J$ that

$$
0 \leq x(\alpha(t)) \leq r \quad \text { for } t \in J
$$

Consequently, for any $t \in J$ and $x \in K \cap \partial \Omega_{r}$, (13) and (22) imply

$$
\begin{aligned}
(T x)(t) & =\int_{0}^{1} H(t, s) q(s) \omega(s) f(s, x(\alpha(s))) d s \\
& \leq H^{0} e^{M} \int_{0}^{1} \omega(s) \frac{1}{e^{M} \gamma H^{0}} x(\alpha(s)) d s \\
& \leq \frac{1}{\gamma} \int_{0}^{1} \omega(s) d s\|x\| \\
& =\|x\|
\end{aligned}
$$

which implies

$$
\|T x\| \leq\|x\|, \quad \forall x \in K \cap \partial \Omega_{r} .
$$

Next, turning to $f_{\infty}=\infty$, there exists $R$ satisfying $0<$ $r<R$ such that

$$
f(t, x) \geq \frac{1}{\delta H_{0} \gamma_{1}} x, \quad \forall t \in J, \quad x \geq R .
$$

Since $0 \leq \alpha(t) \leq t \leq \xi$ on $[0, \xi]$, it follows from $x(t) \geq R$ on $J$ that

$$
x(\alpha(t)) \geq R \text { for } t \in[0, \xi] .
$$

Hence, for $x \in K \cap \partial \Omega_{R}$, it follows from Remark 8 and (22) that

$$
\begin{aligned}
(T x)(t) & =\int_{0}^{1} H(t, s) q(s) \omega(s) f(s, x(\alpha(s))) d s \\
& \geq \delta H_{0} \int_{0}^{1} q(s) \omega(s) f(s, x(\alpha(s))) d s \\
& \geq \delta H_{0} \int_{0}^{\xi} \omega(s) f(s, x(\alpha(s))) d s \\
& \geq \delta H_{0} \int_{0}^{\xi} \omega(s) \frac{1}{\delta H_{0} \gamma_{1}} x(\alpha(s)) d s \\
& \geq \frac{1}{\delta \gamma_{1}} \int_{0}^{\xi} \omega(s) d s \delta\|x\| \\
& =\|x\|,
\end{aligned}
$$

which implies

$$
\|T x\| \geq\|x\|, \quad \forall x \in K \cap \partial \Omega_{R} .
$$

Thus, by (i) of Lemma 13, it follows that $T$ has a fixed point $x$ in $K \cap\left(\bar{\Omega}_{R} \backslash \Omega_{r}\right)$ with

$$
r \leq\|x\| \leq R
$$

Remark 12 shows that problem (3) has at least one positive solution $x$ with

$$
r \leq\|x\| \leq R
$$


Next, we consider the case $f_{0}=\infty$ and $f^{\infty}=0$. Since $f_{0}=\infty$, we can choose $r>0$ such that

$$
f(t, x) \geq \frac{1}{\delta H_{0} \gamma_{1}} x, \quad \forall t \in J, 0 \leq x \leq r .
$$

Since $0 \leq \alpha(t) \leq t \leq \xi$ on $[0, \xi]$, it follows from $0 \leq x(t) \leq$ $r$ on $J$ that

$$
0 \leq x(\alpha(t)) \leq r \quad \text { for } t \in[0, \xi] .
$$

Consequently, for $x \in K \cap \partial \Omega_{r}$, it follows from Remark 8 and (22) that

$$
\begin{aligned}
(T x)(t) & =\int_{0}^{1} H(t, s) q(s) \omega(s) f(s, x(\alpha(s))) d s \\
& \geq \delta H_{0} \int_{0}^{1} q(s) \omega(s) f(s, x(\alpha(s))) d s \\
& \geq \delta H_{0} \int_{0}^{\xi} \omega(s) f(s, x(\alpha(s))) d s \\
& \geq \delta H_{0} \int_{0}^{\xi} \omega(s) \frac{1}{\delta H_{0} \gamma_{1}} x(\alpha(s)) d s \\
& \geq \frac{1}{\delta \gamma_{1}} \int_{0}^{\xi} \omega(s) d s \delta\|x\| \\
& =\|x\|,
\end{aligned}
$$

which implies

$$
\|T x\| \geq\|x\|, \quad \forall x \in K \cap \partial \Omega_{r} .
$$

If $f^{\infty}=0$, we can choose $0<\varepsilon<1 / H^{0} \gamma e^{M}$ and $l>0$ such that

$$
f(t, x) \leq \varepsilon x \quad \text { for } t \in J, x \geq l .
$$

Letting $\zeta=\max _{t \in J, x \in[0, l]} f(t, x)$, then

$$
0 \leq f(t, x) \leq \varepsilon x+\zeta \text { for } t \in J, x \in[0, \infty) .
$$

Since $0 \leq \alpha(t) \leq t \leq 1$ on $J$, it follows from $x(t) \geq l$ or $0 \leq x(t) \leq l$ on $J$ that

$$
x(\alpha(t)) \geq l \quad \text { or } 0 \leq x(\alpha(t)) \leq l \text { for } t \in J .
$$

Let $R \geq \max \left\{2 r, e^{M} H^{0} \gamma \zeta /\left(1-e^{M} H^{0} \gamma \varepsilon\right)\right\}$. Then, for $t \in J$ and $x \in K \cap \partial \Omega_{R}$, (13) and (22) imply

$$
\begin{aligned}
(T x)(t) & =\int_{0}^{1} H(t, s) q(s) \omega(s) f(s, x(\alpha(s))) d s \\
& \leq H^{0} e^{M} \int_{0}^{1} \omega(s) f(s, x(\alpha(s))) d s \\
& \leq H^{0} e^{M} \int_{0}^{1} \omega(s)(\varepsilon x(\alpha(s))+\zeta) d s \\
& \leq H^{0} e^{M} \int_{0}^{1} \omega(s)(\varepsilon\|x\|+\zeta) d s \\
& \leq H^{0} e^{M} \gamma(\varepsilon R+\zeta) \\
& \leq R,
\end{aligned}
$$

which implies

$$
\|T x\| \leq\|x\|, \quad \forall x \in K \cap \partial \Omega_{R} .
$$

Thus, by (ii) of Lemma 13, it follows from (41) and (46) that $T$ has a fixed point $x$ in $K \cap\left(\bar{\Omega}_{R} \backslash \Omega_{r}\right)$ with $r \leq\|x\|<R$. Remark 12 shows that problem (3) has at least one positive solution $x$ with $r \leq\|x\|<R$. This gives the proof of Theorem 14.

4.2. For the Case $i_{0}=0$ and $i_{\infty}=0$. In this subsection, we discuss the existence for the positive solutions of problem (3) under $i_{0}=0$ and $i_{\infty}=0$. For convenience, we introduce the following notation:

$$
f_{0}^{\rho}=\max \left\{\max _{t \in J} \frac{f(t, x)}{\rho}: x \in[0, \rho]\right\} .
$$

Now, we will state and prove the following main result.

Theorem 15. Suppose $\left(H_{1}\right)-\left(H_{3}\right)$ hold. In addition, let the following two conditions hold:

$\left(H_{4}\right)$ there exists $\rho_{1}>0$ such that $f_{0}^{\rho_{1}} \leq 1 / e^{M} H^{0} \gamma$;

$\left(H_{5}\right)$ there exist $\eta>0$ and $\rho_{2}>0$ such that $f(t, x) \geq \eta$ for $t \in J, x \geq \rho_{2}$; furthermore, $\rho_{1} \neq \rho_{2}$.

Then problem (3) has at least one positive solution.

Proof. Without loss of generality, we may assume that $\rho_{1}<\rho_{2}$. Considering $f_{0}^{\rho_{1}} \leq 1 / e^{M} H^{0} \gamma$, we have $f(t, x) \leq\left(1 / e^{M} H^{0} \gamma\right) \rho_{1}$ for $0 \leq x \leq \rho_{1}, t \in J$.

Since $0 \leq \alpha(t) \leq t \leq 1$ on $J$, it follows from $0 \leq x(t) \leq \rho_{1}$ on $J$ that

$$
0 \leq x(\alpha(t)) \leq \rho_{1} .
$$

Consequently, for any $t \in J$ and $y \in K \cap \partial \Omega_{\rho_{1}}$, (13) and (22) imply

$$
\begin{aligned}
(T x)(t) & =\int_{0}^{1} H(t, s) q(s) \omega(s) f(s, x(\alpha(s))) d s \\
& \leq H^{0} e^{M} \int_{0}^{1} \frac{1}{H^{0} \gamma e^{M}} \rho_{1} \omega(s) d s \\
& =\rho_{1},
\end{aligned}
$$

which implies

$$
\|T x\| \leq\|x\|, \quad \forall x \in K \cap \partial \Omega_{\rho} .
$$

On the other hand, from $\left(H_{5}\right)$, when a $\rho_{2}$ is fixed, then there exists a $\eta>0$ such that

$$
f(t, x) \geq \eta \geq \frac{\rho_{2}}{\delta H_{0} \gamma_{1}}
$$

for $t \in J$ and $x \geq \rho_{2}$. Since $0 \leq \alpha(t) \leq t \leq \xi$ on $[0, \xi]$, it follows from $x(t) \geq \rho_{2}$ on $J$ that

$$
x(\alpha(t)) \geq \rho_{2} .
$$


Hence, for $x \in K \cap \partial \Omega_{\rho_{2}}$, it follows from Remark 8 and (22) that

$$
\begin{aligned}
(T x)(t) & =\int_{0}^{1} H(t, s) q(s) \omega(s) f(s, x(\alpha(s))) d s \\
& \geq \min _{t \in[0, \xi]} \int_{0}^{1} H(t, s) q(s) \omega(s) f(s, x(\alpha(s))) d s \\
& \geq \delta H_{0} \int_{0}^{1} q(s) \omega(s) f(s, x(\alpha(s))) d s \\
& \geq \delta H_{0} \int_{0}^{\xi} \omega(s) f(s, x(\alpha(s))) d s \\
& \geq \delta \eta H_{0} \int_{0}^{\xi} \omega(s) d s \\
& =\rho_{2},
\end{aligned}
$$

which implies

$$
\|T x\| \geq\|x\|, \quad \forall x \in K \cap \partial \Omega_{\rho_{2}} .
$$

Thus, by (i) of Lemma 13, it follows that $T$ has a fixed point $x$ in $K \cap\left(\bar{\Omega}_{\rho_{2}} \backslash \Omega_{\rho_{1}}\right)$ with

$$
\rho_{1} \leq\|x\| \leq \rho_{2} .
$$

Thus, it follows from Remark 12 that problem (3) has at least one positive solution $x$ with $\rho_{1} \leq\|x\| \leq \rho_{2}$. This finishes the proof of Theorem 15.

We remark that condition $\left(\mathrm{H}_{4}\right)$ in Theorem 15 can be replaced by the following condition:

$$
\left(H_{4}\right)^{\prime} f^{0} \leq 1 / H^{0} \gamma e^{M},
$$

which is a special case of $\left(H_{5}\right)$.

Corollary 16. Suppose $\left(H_{1}\right)-\left(H_{3}\right),\left(H_{4}\right)^{\prime}$, and $\left(H_{5}\right)$ hold. Then problem (3) has at least one positive solution.

Proof. We show that $\left(H_{4}\right)^{\prime}$ implies $\left(H_{4}\right)$. Suppose that $\left(H_{4}\right)^{\prime}$ holds. Then, there exists a positive number $\rho_{1} \neq \rho_{2}$ such that

$$
\frac{f(t, x)}{x} \leq \frac{1}{H^{0} \gamma e^{M}}, \quad t \in J, 0<x \leq \rho_{1} .
$$

Hence, we obtain

$$
f(t, x) \leq \frac{1}{H^{0} \gamma e^{M}} x \leq \frac{1}{H^{0} \gamma e^{M}} \rho_{1}, \quad t \in J, 0<x \leq \rho_{1} .
$$

Therefore, $\left(H_{4}\right)$ holds. Hence, by Theorem 15, problem (3) has at least one positive solution.

Theorem 17. Suppose $\left(H_{1}\right)-\left(H_{4}\right)$ hold. In addition, let the following condition hold:

$$
\left(H_{6}\right) f_{\infty} \geq 1 / \delta H_{0} \gamma_{1} \text {. }
$$

Then problem (3) has at least one positive solution.
Proof. The proof is similar to that of (50) and (35), respectively.

Corollary 18. Suppose $\left(H_{1}\right)-\left(H_{3}\right),\left(H_{4}\right)^{\prime}$, and $\left(H_{6}\right)$ hold. Then problem (3) has at least one positive solution.

4.3. For the Case $i_{0}=1$ and $i_{\infty}=0$ or $i_{0}=0$ and $i_{\infty}=1$. In this subsection, we discuss the existence for the positive solutions of problem (3) for the case $i_{0}=1$ and $i_{\infty}=0$ or $i_{0}=0$ and $i_{\infty}=1$. For convenience, we introduce the following notation:

$$
l=\frac{1}{H^{0} \gamma e^{M}}, \quad L=\frac{1}{\delta^{2} H_{0} \gamma_{1}} .
$$

Theorem 19. Suppose $\left(H_{1}\right)-\left(H_{3}\right)$ hold, $f^{0} \in[0, l)$, and $f_{\infty} \in$ $(L, \infty)$. Then problem (3) has at least one positive solution.

Proof. The proof is similar to that of Theorem 15.

Theorem 20. Suppose $\left(H_{1}\right)-\left(H_{3}\right)$ hold, $f_{0} \in(L, \infty)$, and $f^{\infty} \in[0, l)$. Then problem (3) has at least one positive solution.

Proof. Considering $f_{0} \in(L, \infty)$, then there exists $\rho_{1}>0$ such that $f(t, x)>L x$ for $0 \leq x \leq \rho_{1}, t \in J$.

Since $0 \leq \alpha(t) \leq t \leq 1$ on $J$, it follows from $0 \leq x(t) \leq \rho_{1}$ on $J$ that

$$
0 \leq x(\alpha(t)) \leq \rho_{1} .
$$

Consequently, for $x \in K \cap \partial \Omega_{\rho_{1}}$, it follows from Remark 8 and (22) that

$$
\begin{aligned}
(T x)(t) & =\int_{0}^{1} H(t, s) q(s) \omega(s) f(s, x(\alpha(s))) d s \\
& \geq \min _{t \in[0, \xi]} \int_{0}^{1} H(t, s) q(s) \omega(s) f(s, x(\alpha(s))) d s \\
& \geq \delta H_{0} \int_{0}^{1} q(s) \omega(s) f(s, x(\alpha(s))) d s \\
& \geq \delta H_{0} \int_{0}^{\xi} \omega(s) f(s, x(\alpha(s))) d s \\
& \geq \delta H_{0} \int_{0}^{\xi} \omega(s) L x(\alpha(s)) d s \\
& \geq \delta H_{0} \int_{0}^{\xi} \omega(s) L \delta\|x\| d s \\
& \geq\|x\|,
\end{aligned}
$$

which shows

$$
\|T x\| \geq\|x\|, \quad \forall x \in K \cap \partial \Omega_{\rho} .
$$

Next, we turn to $f^{\infty} \in[0, l)$. In fact, we can show that $f^{\infty} \in[0, l)$ implies $\left(H_{4}\right)$. 
Let $\tau \in\left(f^{\infty}, l\right)$. Then, there exists $r>\tau$ such that $\max _{t \in J} f(t, x) \leq \tau x$ for $y \in[r, \infty)$. Let

$$
\begin{gathered}
\beta=\max \left\{\max _{t \in J} f(t, x): 0 \leq x \leq r\right\}, \\
\rho_{1}^{*}>\max \left\{\frac{\beta}{l-\tau}, \rho\right\} .
\end{gathered}
$$

Then, we have

$$
\max _{0 \leq t \leq 1} f(t, x) \leq \tau x+\beta \leq \tau \rho_{1}^{*}+\beta<l \rho_{1}^{*}, \quad \forall x \in\left[0, \rho_{1}^{*}\right] .
$$

This implies that $f_{0}^{\rho_{1}^{*}} \leq l$. Hence, $f^{\infty} \in[0, l)$ implies that $\left(\mathrm{H}_{4}\right)$.

Similar to the proof of (46), we have

$$
\|T x\| \leq\|x\|, \quad \forall x \in K \cap \partial \Omega_{\rho^{*}} .
$$

Thus, by (ii) of Lemma 13, it follows that $T$ has a fixed point $x$ in $K \cap\left(\bar{\Omega}_{\rho_{1}^{*}} \backslash \Omega_{\rho}\right)$ with

$$
\rho \leq\|x\| \leq \rho_{1}^{*} .
$$

This finishes the proof of Theorem 20 .

From Theorems 19 and 20, we have the following result.

Corollary 21. Assume that $\left(\mathrm{H}_{1}\right)-\left(\mathrm{H}_{3}\right)$ hold. Furthermore, suppose that $f^{0}=0$ and condition $\left(H_{5}\right)$ in Theorem 15 hold. Then problem (3) has at least one positive solution.

Theorem 22. Suppose $\left(H_{1}\right)-\left(H_{3}\right)$ hold, $f^{0} \in(0, l)$, and $f_{\infty}=$ $\infty$. Then problem (3) has at least one positive solution.

Proof. The proof is similar to that of Theorem 15.

Theorem 23. Suppose $\left(H_{1}\right)-\left(H_{3}\right)$ hold, $f_{0}=\infty$, and $f^{\infty} \in$ $(0, l)$. Then problem (3) has at least one positive solution.

Proof. The proof is similar to that of Theorem 15.

From Theorems 22 and 23, the following corollaries are easily obtained.

Corollary 24. Assume that $\left(\mathrm{H}_{1}\right)-\left(\mathrm{H}_{3}\right)$ hold. Furthermore, suppose that $f_{0}=\infty$ and condition $\left(H_{4}\right)$ in Theorem 15 hold. Then problem (3) has at least one positive solution.

Corollary 25. Assume that $\left(\mathrm{H}_{1}\right)-\left(\mathrm{H}_{3}\right)$ hold. Furthermore, suppose that $f_{\infty}=\infty$ and condition $\left(\mathrm{H}_{4}\right)$ in Theorem 15 hold. Then problem (3) has at least one positive solution.

4.4. For the Case $i_{0}=0$ and $i_{\infty}=2$ or $i_{0}=2$ and $i_{\infty}=0$. In this subsection, we study the existence of multiple positive solutions for the problem (3) for the case $i_{0}=0$ and $i_{\infty}=2$ or $i_{0}=2$ and $i_{\infty}=0$.

Combining the proof of Theorems 14 and 15, the following theorem is easily proven.
Theorem 26. Suppose that $\left(H_{1}\right)-\left(H_{3}\right), i_{0}=0$, and $i_{\infty}=2$, and the condition $\left(\mathrm{H}_{4}\right)$ of Theorem 15 hold. Then problem (3) has at least two positive solutions.

Corollary 27. Suppose that $\left(H_{1}\right)-\left(H_{3}\right), i_{0}=0$, and $i_{\infty}=2$, and the condition $\left(\mathrm{H}_{4}\right)^{\prime}$ of Corollary 16 hold. Then problem (3) has at least two positive solutions.

Theorem 28. Suppose that $\left(H_{1}\right)-\left(H_{3}\right), i_{0}=2$, and $i_{\infty}=0$, and the condition $\left(\mathrm{H}_{5}\right)$ of Theorem 15 hold. Then problem (3) has at least two positive solutions.

Corollary 29. Suppose that $\left(H_{1}\right)-\left(H_{3}\right), i_{0}=2$, and $i_{\infty}=0$, and the condition $\left(\mathrm{H}_{6}\right)$ of Theorem 17 hold. Then problem (3) has at least two positive solutions.

\section{Three Examples}

To illustrate how our main results can be used in practice, we present three examples.

Example 1. Consider the following boundary value problem:

$$
\begin{gathered}
-x^{\prime \prime}(t)+b x(t)=\omega(t) f(t, x(\alpha(t))), \quad t \in J, \\
x^{\prime}(0)=0, \quad x(1)=\int_{0}^{1} x(t) d t,
\end{gathered}
$$

where $\alpha \in C(J, J), \alpha(t) \leq t$ on $J$, and

$$
\omega(t)=\frac{1}{\sqrt{t}}, \quad f(t, x)=\sqrt[n]{1+t^{n}} x^{n} ;
$$

here $n \geq 2$ is a positive integral number.

This means that problem (66) involves the advanced argument $\alpha$. For example, we can take $\alpha(t)=t^{2}$. It is clear that $\omega$ may be singular at $t=0$ and/or $t=1$ and $f$ is both nonnegative and continuous.

Problem (66) can be regarded as a problem of the form (3), where $a(t) \equiv 0, b(t) \equiv 1$, and $h(t) \equiv 1$.

Let $\phi$ and $\psi$ satisfy

$$
\begin{array}{lll}
L \phi=0, & \phi^{\prime}(0)=0, & \phi(1)=1, \\
L \psi=0, & \psi(0)=1, & \psi(1)=0,
\end{array}
$$

where $L x=-x^{\prime \prime}+x(t)$ and

$$
\begin{gathered}
\phi(t)=\frac{e^{1-t}+e^{1+t}}{1+e^{2}}, \quad \phi(0)=\frac{2 e}{1+e^{2}}, \\
\psi(t)=\frac{-e^{2-t}+e^{t}}{1-e^{2}}, \\
\psi^{\prime}(0)=\frac{e^{2}+1}{1-e^{2}}, \quad q(t)=1, \\
\Delta:=-\phi(0) \psi^{\prime}(0)=\frac{2 e}{e^{2}-1}>0 .
\end{gathered}
$$

It follows from the definition of $\omega, f$, and $h$ that $\left(H_{1}\right)$ $\left(\mathrm{H}_{3}\right)$ hold, and

$$
f^{0}=0, \quad f_{\infty}=\infty .
$$


Hence, by Theorem 14, problem (66) has at least one positive solution.

Example 2. If we replace $f(t, x)$ in Example 1 by

$$
f(t, x)=\left(\frac{1}{3}+\frac{1}{3} t\right)+2 \sin x
$$

in this case, by Theorem 20, we obtain that if $L<7 / 3$ and $l>$ $2 / 3$, then problem (66) admits at least one positive solution.

In fact, we can prove that

$$
\begin{aligned}
f_{0} & =\liminf _{x \rightarrow 0} \min _{t \in J} \frac{f(t, x)}{x}=\frac{7}{3}, \\
f^{\infty} & =\limsup _{x \rightarrow \infty} \max _{t \in J} \frac{f(t, x)}{x}=\frac{2}{3},
\end{aligned}
$$

which shows that $f_{0} \in(L, \infty)$ and $f^{\infty} \in[0, l)$.

Example 3. If we replace $f(t, x)$ in Example 1 by

$$
f(t, x)=(1+t) x^{2}+x^{1 / 2},
$$

in this case, by Theorem 26, we obtain that problem (66) admits at least two positive solutions.

In fact, we can prove that

$$
\begin{aligned}
& f_{0}=\liminf _{x \rightarrow 0} \min _{t \in J} \frac{f(t, x)}{x}=\infty, \\
& f_{\infty}=\limsup _{x \rightarrow \infty} \max _{t \in J} \frac{f(t, x)}{x}=\infty,
\end{aligned}
$$

which shows that $i_{0}=0$ and $i_{\infty}=2$.

\section{Conflict of Interests}

The authors declare that there is no conflict of interests regarding the publication of this paper.

\section{Acknowledgments}

The authors are indebted to the referee's suggestions. These have greatly improved this paper. This work is sponsored by the Project NSFC (11301178, 11171032), the Fundamental Research Funds for the Central Universities (2014MS58), and the Improving Project of Graduate Education of Beijing Information Science and Technology University (YJT201416).

\section{References}

[1] J. R. Cannon, "The solution of the heat equation subject to the specification of energy," Quarterly of Applied Mathematics, vol. 21, pp. 155-160, 1963.

[2] N. I. Ionkin, "Solution of a boundary value problem in heat conduction theory with nonlocal boundary conditions," Differential Equations, vol. 13, pp. 294-304, 1977.

[3] R. Y. Chegis, "Numerical solution of a heat conduction problem with an integral boundary condition," Lietuvos Matematikos Rinkinys, vol. 24, pp. 209-215, 1984.
[4] E. H. Doha, A. H. Bhrawy, and R. M. Hafez, "On shifted Jacobi spectral method for high-order multi-point boundary value problems," Communications in Nonlinear Science and Numerical Simulation, vol. 17, no. 10, pp. 3802-3810, 2012.

[5] R. Y. Ma, "Existence of positive solutions for a nonlinear $m$ point boundary value problem," Acta Mathematica Sinica, vol. 46, no. 4, pp. 785-794, 2003.

[6] Z. Bai and Z. Du, "Positive solutions for some second-order four-point boundary value problems," Journal of Mathematical Analysis and Applications, vol. 330, no. 1, pp. 34-50, 2007.

[7] R. I. Avery, C. J. Chyan, and J. Henderson, "Twin solutions of boundary value problems for ordinary differential equations and finite difference equations," Computers \& Mathematics with Applications, vol. 42, no. 3-5, pp. 695-704, 2001.

[8] Y. Zhao and H. Chen, "Existence of multiple positive solutions for $m$-point boundary value problems in Banach spaces," Journal of Computational and Applied Mathematics, vol. 215, no. 1, pp. 79-90, 2008.

[9] Y. Liu and D. O’Regan, "Multiplicity results for a class of fourth order semipositone $m$-point boundary value problems," Applicable Analysis, vol. 91, no. 5, pp. 911-921, 2012.

[10] Z. Du and L. Kong, "Asymptotic solutions of singularly perturbed second-order differential equations and application to multi-point boundary value problems," Applied Mathematics Letters, vol. 23, no. 9, pp. 980-983, 2010.

[11] C. P. Gupta, "A generalized multi-point boundary value problem for second order ordinary differential equations," Applied Mathematics and Computation, vol. 89, no. 1-3, pp. 133-146, 1998.

[12] M. Feng and W. Ge, "Positive solutions for a class of $m$ point singular boundary value problems," Mathematical and Computer Modelling, vol. 46, no. 3-4, pp. 375-383, 2007.

[13] B. Ahmad, A. Alsaedi, and B. S. Alghamdi, "Analytic approximation of solutions of the forced Duffing equation with integral boundary conditions," Nonlinear Analysis, Real World Applications, vol. 9, no. 4, pp. 1727-1740, 2008.

[14] G. Infante and J. R. L. Webb, "Nonlinear non-local boundaryvalue problems and perturbed Hammerstein integral equations," Proceedings of the Edinburgh Mathematical Society, vol. 49, no. 3, pp. 637-656, 2006.

[15] L. Kong, "Second order singular boundary value problems with integral boundary conditions," Nonlinear Analysis: Theory, Methods \& Applications, vol. 72, no. 5, pp. 2628-2638, 2010.

[16] X. Zhang and M. Feng, "Existence and dependence of positive solution on parameter for the one-dimensional singular $p$ Laplacian," Journal of Mathematical Analysis and Applications, vol. 413, pp. 566-582, 2014.

[17] X. Zhang and M. Feng, "Transformation techniques and fixed point theories to establish the positive solutions of second order impulsive differential equations," Journal of Computational and Applied Mathematics, vol. 271, pp. 117-129, 2014.

[18] A. Boucherif, "Second-order boundary value problems with integral boundary conditions," Nonlinear Analysis: Theory, Methods \& Applications, vol. 70, no. 1, pp. 364-371, 2009.

[19] M. Feng, D. Ji, and W. Ge, "Positive solutions for a class of boundary-value problem with integral boundary conditions in Banach spaces," Journal of Computational and Applied Mathematics, vol. 222, no. 2, pp. 351-363, 2008.

[20] C. Yang, C. Zhai, and J. Yan, "Positive solutions of the threepoint boundary value problem for second order differential equations with an advanced argument," Nonlinear Analysis: 
Theory, Methods \& Applications, vol. 65, no. 10, pp. 2013-2023, 2006.

[21] T. Jankowski, "Solvability of three point boundary value problems for second order differential equations with deviating arguments," Journal of Mathematical Analysis and Applications, vol. 312, no. 2, pp. 620-636, 2005.

[22] T. Jankowski, "Nonnegative solutions to nonlocal boundary value problems for systems of second-order differential equations dependent on the first-order derivatives," Nonlinear Analysis, vol. 87, pp. 83-101, 2013.

[23] T. Jankowski, "Positive solutions to second-order differential equations with dependence on the first-order derivative and nonlocal boundary conditions," Boundary Value Problems, vol. 2013, article 8, 2013.

[24] D. Jiang and J. Wei, "Monotone method for first- and secondorder periodic boundary value problems and periodic solutions of functional differential equations," Nonlinear Analysis, vol. 50, no. 7, Ser. A: Theory Methods, pp. 885-898, 2002.

[25] G. Wang, "Monotone iterative technique for boundary value problems of a nonlinear fractional differential equation with deviating arguments," Journal of Computational and Applied Mathematics, vol. 236, no. 9, pp. 2425-2430, 2012.

[26] G. Wang, L. Zhang, and G. Song, "Systems of first order impulsive functional differential equations with deviating arguments and nonlinear boundary conditions," Nonlinear Analysis: Theory, Methods \& Applications, vol. 74, no. 3, pp. 974-982, 2011.

[27] C. Hu, B. Liu, and S. Xie, "Monotone iterative solutions for nonlinear boundary value problems of fractional differential equation with deviating arguments," Applied Mathematics and Computation, vol. 222, pp. 72-81, 2013.

[28] D. Bai and Y. Xu, "Positive solutions of second-order two-delay differential systems with twin-parameter," Nonlinear Analysis: Theory, Methods \& Applications, vol. 63, no. 4, pp. 601-617, 2005.

[29] B. Du, X. Hu, and W. Ge, "Positive solutions to a type of multipoint boundary value problem with delay and one-dimensional p-Laplacian," Applied Mathematics and Computation, vol. 208, no. 2, pp. 501-510, 2009.

[30] D. Jiang, "Multiple positive solutions for boundary value problems of second-order delay differential equations," Applied Mathematics Letters, vol. 15, no. 5, pp. 575-583, 2002.

[31] W. Wang and J. Shen, "Positive solutions to a multi-point boundary value problem with delay," Applied Mathematics and Computation, vol. 188, no. 1, pp. 96-102, 2007.

[32] Y. Wang, W. Zhao, and W. Ge, "Multiple positive solutions for boundary value problems of second order delay differential equations with one-dimensional $p$-Laplacian," Journal of Mathematical Analysis and Applications, vol. 326, no. 1, pp. 641-654, 2007.

[33] T. Jankowski, "Positive solutions for fourth-order differential equations with deviating arguments and integral boundary conditions," Nonlinear Analysis, vol. 73, no. 5, pp. 1289-1299, 2010.

[34] T. Jankowski and R. Jankowski, "Multiple solutions of boundary-value problems for fourth-order differential equations with deviating arguments," Journal of Optimization Theory and Applications, vol. 146, no. 1, pp. 105-115, 2010.

[35] M. Feng, X. Zhang, and X. Yang, "Positive solutions of nthorder nonlinear impulsive differential equation with nonlocal boundary conditions," Boundary Value Problems, vol. 2011, article 19, Article ID 456426, 2011.

[36] D. J. Guo and V. Lakshmikantham, Nonlinear Problems in Abstract Cones, Academic Press, New York, NY, USA, 1988.
[37] H. Wang, "Positive periodic solutions of functional differential equations," Journal of Differential Equations, vol. 202, no. 2, pp. 354-366, 2004.

[38] H. Sun and W. Li, "Existence theory for positive solutions to one-dimensional $p$-Laplacian boundary value problems on time scales," Journal of Differential Equations, vol. 240, no. 2, pp. 217-248, 2007. 


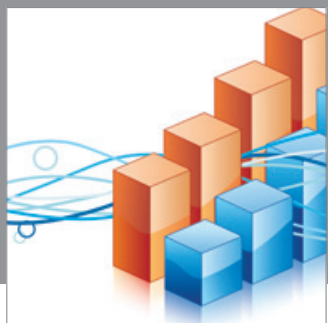

Advances in

Operations Research

mansans

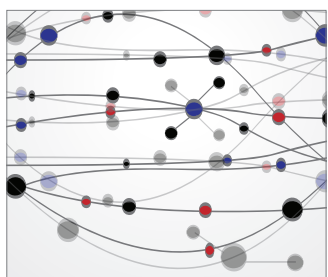

The Scientific World Journal
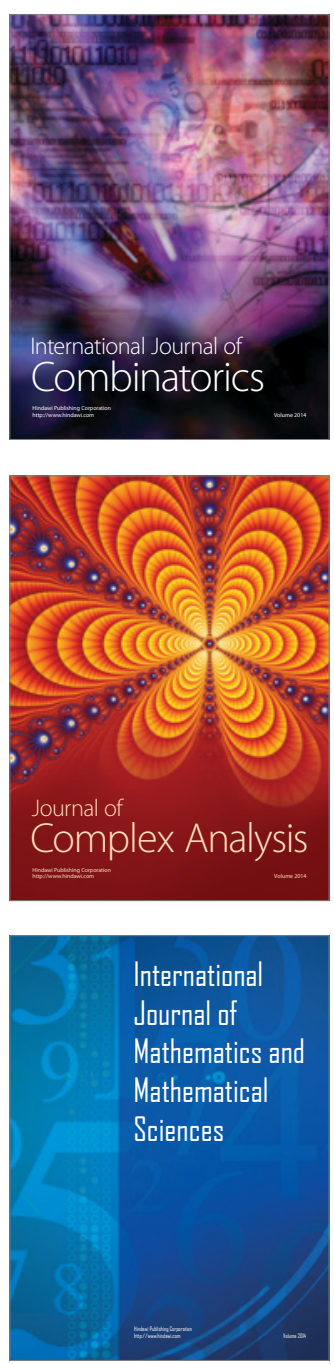
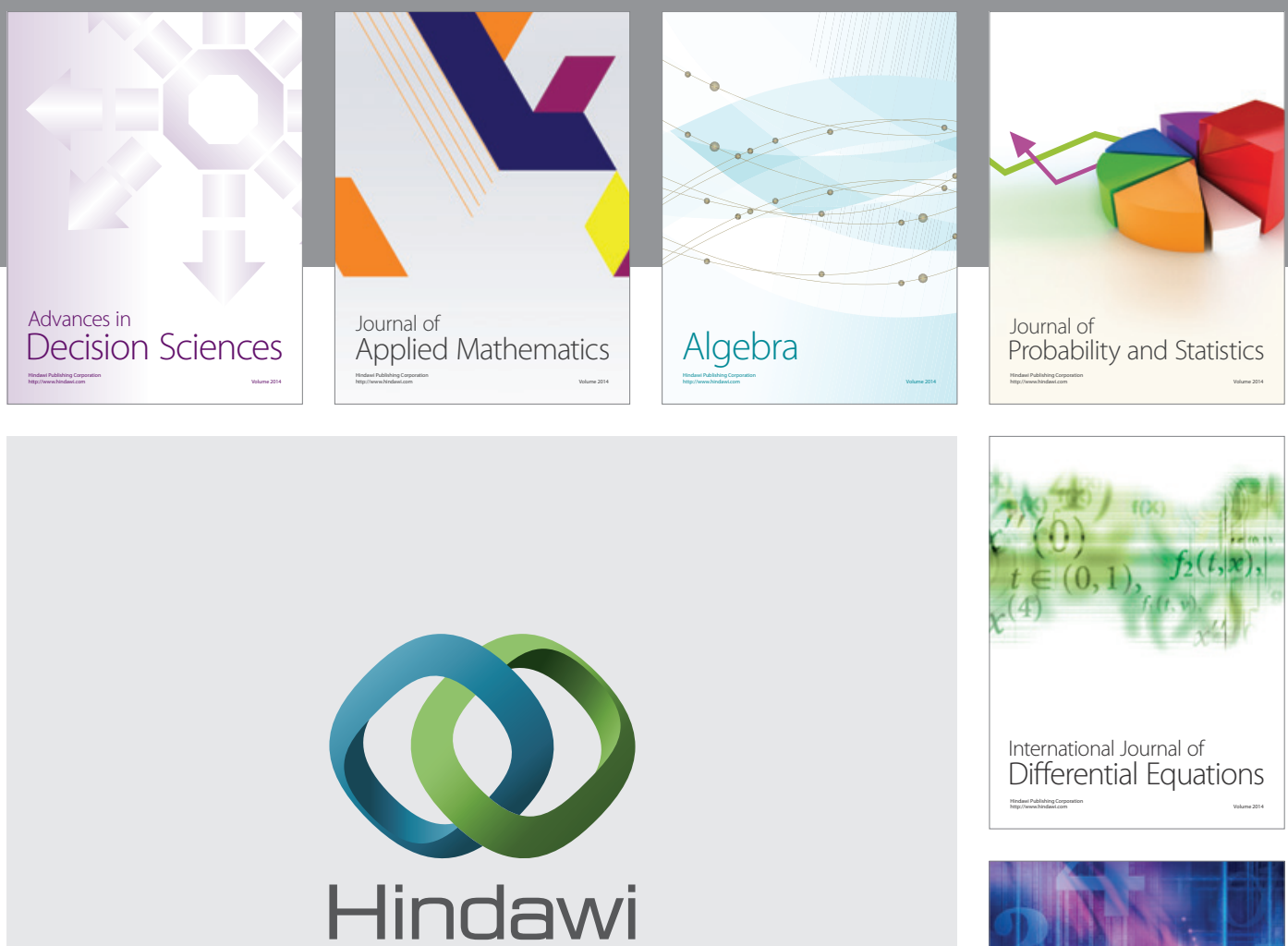

Submit your manuscripts at http://www.hindawi.com
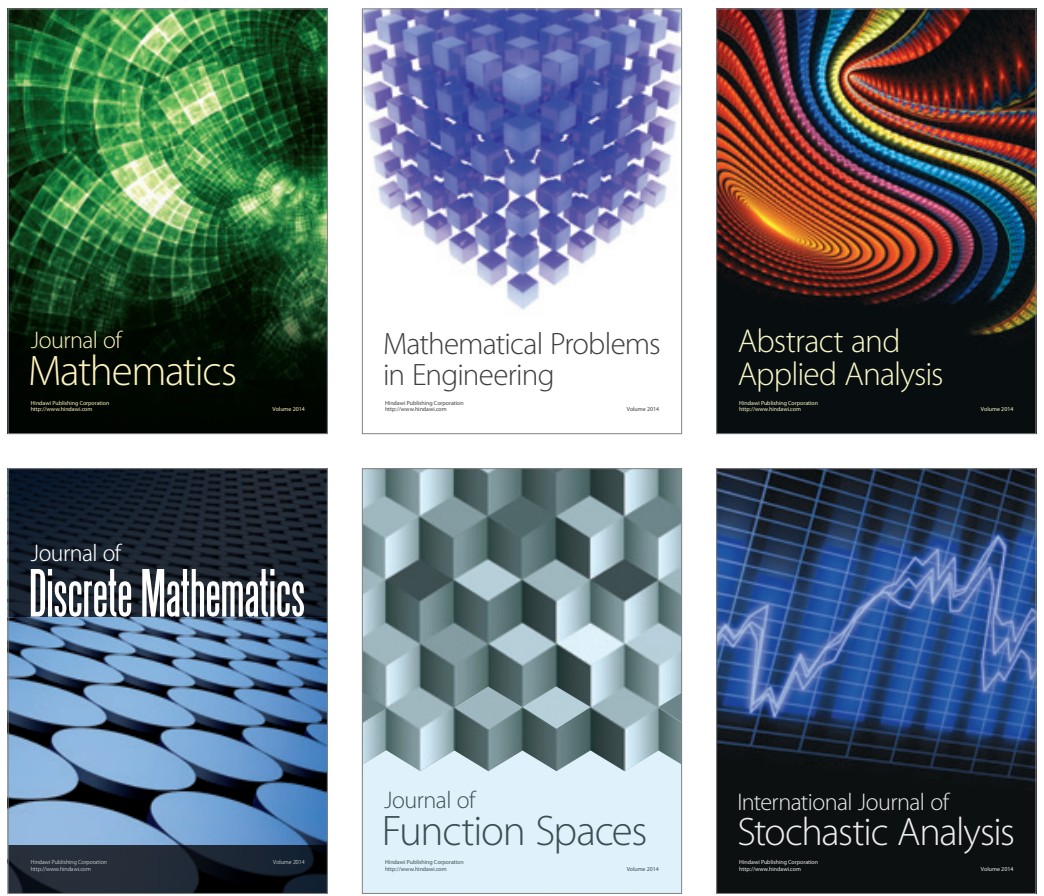

Journal of

Function Spaces

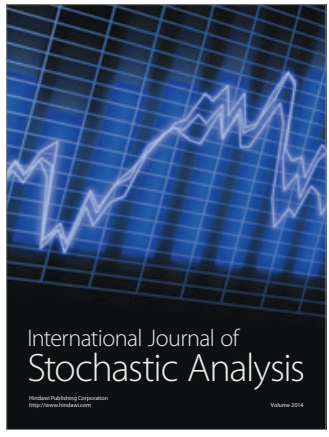

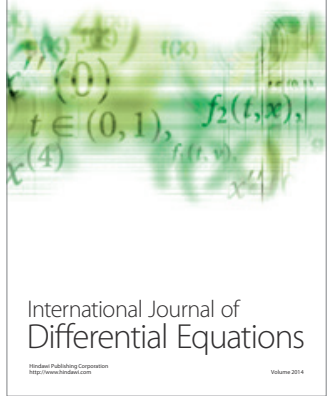
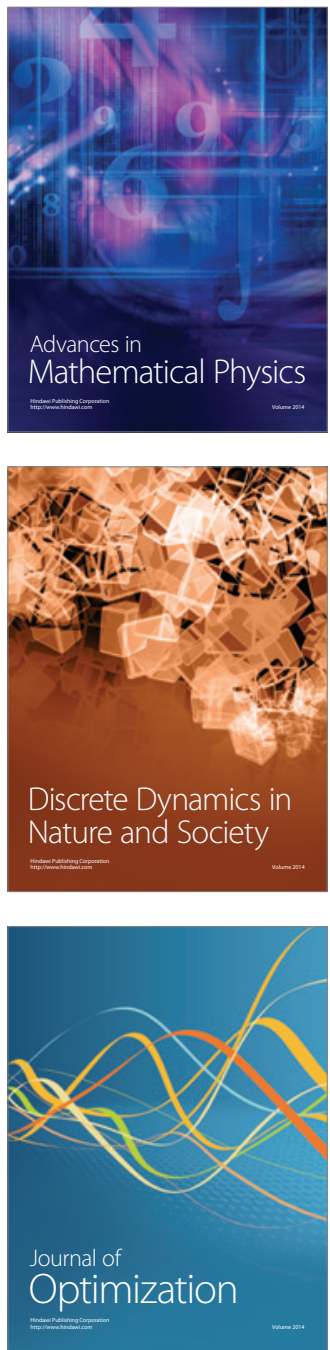